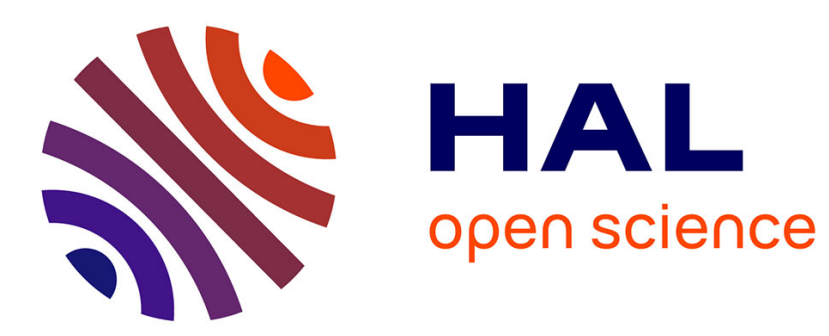

\title{
Geometry and the entropic cost of locally favoured structures in a liquid \\ Pierre Ronceray, Peter Harrowell
}

\section{To cite this version:}

Pierre Ronceray, Peter Harrowell. Geometry and the entropic cost of locally favoured structures in a liquid. Journal of Chemical Physics, 2012, 136 (13), pp.134504. 10.1063/1.3701617 . hal-03407984

\section{HAL Id: hal-03407984 \\ https://hal.science/hal-03407984}

Submitted on 28 Oct 2021

HAL is a multi-disciplinary open access archive for the deposit and dissemination of scientific research documents, whether they are published or not. The documents may come from teaching and research institutions in France or abroad, or from public or private research centers.
L'archive ouverte pluridisciplinaire HAL, est destinée au dépôt et à la diffusion de documents scientifiques de niveau recherche, publiés ou non, émanant des établissements d'enseignement et de recherche français ou étrangers, des laboratoires publics ou privés. 


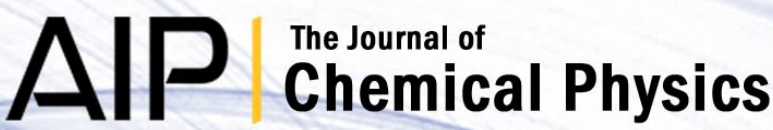

\section{Geometry and the entropic cost of locally favoured structures in a liquid}

Pierre Ronceray and Peter Harrowell

Citation: J. Chem. Phys. 136, 134504 (2012); doi: 10.1063/1.3701617

View online: http://dx.doi.org/10.1063/1.3701617

View Table of Contents: http://jcp.aip.org/resource/1/JCPSA6/v136/i13

Published by the American Institute of Physics.

\section{Additional information on J. Chem. Phys.}

Journal Homepage: http://jcp.aip.org/

Journal Information: http://jcp.aip.org/about/about_the_journal

Top downloads: http://jcp.aip.org/features/most_downloaded

Information for Authors: http://jcp.aip.org/authors

\section{ADVERTISEMENT}

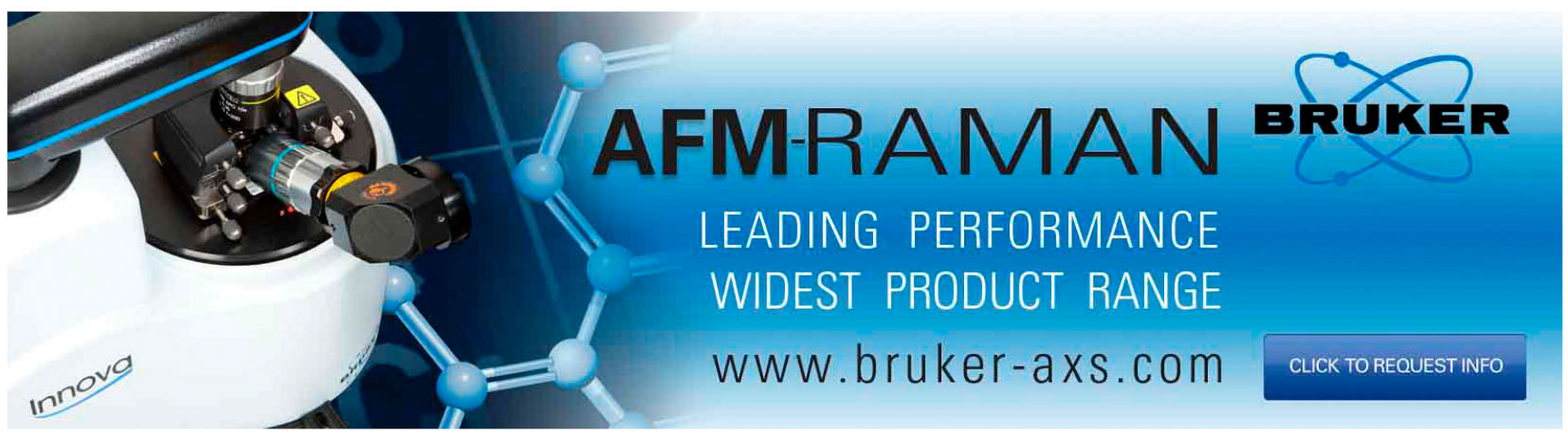




\title{
Geometry and the entropic cost of locally favoured structures in a liquid
}

\author{
Pierre Ronceray ${ }^{1}$ and Peter Harrowell ${ }^{2}$ \\ ${ }^{1}$ École Normale Supérieure, Paris, France \\ ${ }^{2}$ School of Chemistry, University of Sydney, Sydney, New South Wales 2006, Australia
}

(Received 5 February 2012; accepted 21 March 2012; published online 5 April 2012)

\begin{abstract}
The role of the geometry of locally favoured structures in an equilibrium liquid is analyzed within a recently developed lattice model. The local geometry is shown to influence the liquid through the entropy and the associated density of states. We show that favoured local structures with low symmetry will, generally, incur a low entropy cost and, as a consequence, the liquid will exhibit a substantial accumulation of these low energy environments on cooling prior to the freezing transition. (C) 2012 American Institute of Physics. [http://dx.doi.org/10.1063/1.3701617]
\end{abstract}

Faced with the task of describing dense amorphous structure, a fruitful approach has been resolved the global arrangement of particles into the distribution of distinct local coordination geometries. While the organization of these elements over intermediate length scales still represents a challenge, ${ }^{1}$ the energetics of many materials of interest will be dominated by the local structures. A growing number of studies, both simulations ${ }^{2}$ and experimental, ${ }^{3}$ have demonstrated that favoured local structures (FLS's) can, indeed, be identified and are observed to achieve a significant concentration as the temperature is lowered. FLS's have been invoked, in the context of supercooled liquids, to account for the kinetic stability of the supercooled liquid with respect to crystallization ${ }^{4}$ and/or for the non-Arrhenius increase in the structural relaxation time..$^{5}$ In this paper, we shall demonstrate that the geometry of the FLS also exerts an important influence on the equilibrium liquid through its influence on the liquid density of states.

To study the role of favoured local structure, we have developed a simple lattice model, the FLS model, that allows for the direct choice of the stable local structure. Previously ${ }^{6}$ we have described the model, its crystalline ground states and its variety of freezing transitions. The model consists of a lattice populated by two state (Ising) spins for which we can identify all the distinct possible nearest neighbour environments about an arbitrary site. ("Distinct" means that we do not distinguish local spin configurations that are related by a rotation.) In the 2D triangular lattice there are 13 such local structures. Eight of these local structures are sketched in Figure 1, the 5 others being related to one of them by the global spin inversion and therefore have the same properties in this model. We shall select one of these local structures to be "favoured," assigning energy of -1 to every site (irrespective of the sign of the spin on that site) which has the favoured neighbourhood. All other sites are assigned zero energy. We have carried out extensive Monte Carlo simulations of the model over a range of temperatures using a Metropolis weighting ${ }^{7}$ and random flips of individual spins (i.e., Glauber dynamics). The number of sites $N$ is taken equal to $60 \times 60$ in the results presented in this paper and periodic boundaries are imposed.
The temperature dependence of the average energy per site $E(T)$ is plotted in Figure 2 for the different choices of FLS. We have restricted ourselves to temperatures well above the freezing points (see Table I). To make the differences in temperature dependence clearer, we have subtracted the energy for the random spin configuration energy $E_{\infty}$ (corresponding to the $T \rightarrow \infty$ limit). $E_{\infty}$ is simply that fraction of the $2^{6}$ possible local configurations corresponding to the selected FLS, i.e., $E_{\infty}=-g / 2^{6}$, where the multiplicity $g$ is the number of distinct ways that an FLS can be placed on a given site. This number ranges from 1 for the totally symmetric $\{0\}$ structure to 12 for the $\{32\}$ structure which has neither rotational nor reflection symmetry. (Values for $g$ are provided in Table I.) We find a large variation in the temperature dependence of $E$ with the choice of the FLS. Liquids with one of $\{0\},\{22\}$, or $\{31\}$ structures favoured show the weakest decrease in energy on cooling prior to the freezing transition. In contrast, local structures $\{1\}$ or $\{32\}$ exhibit a larger decrease in energy on similar cooling. The energy contribution to the heat capacity per site $C_{V}$ is equal to the temperature derivative of $E$ so that the two sets of FLS's mentioned produce liquids with low and high $C_{V}$, respectively. It is interesting to note that if, instead of the racemic $\{32\}$ structure, we choose a single enantiomer as the favoured local structure (an option designated as $\{32 \mathrm{c}\}$ on Figure 2) then the heat capacity $C_{V}$ is substantially reduced.

Since the energy per FLS is, by construction, independent of its geometry, we need to consider how the local structure influences the entropy to understand the relationship between the FLS and $C_{V}$. The entropy per site $S(E)$ is calculated by integration from the $T \rightarrow \infty$ limit using

$$
S(E)=\ln (2)-\int_{E}^{E_{\infty}} \frac{d E^{\prime}}{T}
$$

and plotted in Figure 2. $S\left(E_{\infty}\right)=\ln (2)$, the entropy of a site occupied by a 2-state spin in the limit of high $T$ (in this paper we take $k_{B}=1$ ). The entropy, when expressed as a function of energy, is simply related to the density of states $\Omega(E)$ (where $\Omega(E) d E$ equals the number of states with energies between $E$ and $E+d E)$ by the relation $S(E)=(1 / N) \ln \Omega(E)$. (To connect this lattice model with off-lattice simulations of interacting particles one should equate the density of states of 


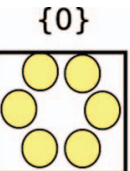

$\{24\}$
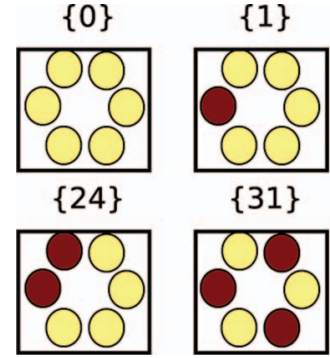

$\{31\}$
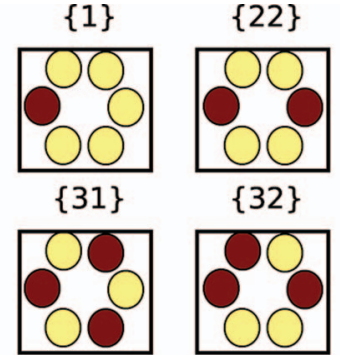

$\{32\}$
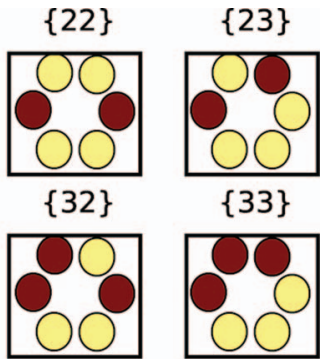

$\{33\}$

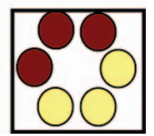

FIG. 1. The 8 distinct local structures available to the FLS model on the triangular lattice. The $\{32\}$ structure being chiral, we will refer to it as $\{32 \mathrm{c}\}$ when only one enantiomer is favoured, and as $\{32\}$ when both are.

the lattice model with the distribution of energies of the local minima in the potential energy surface in the case of particles with continuous degrees of freedom. $\left.{ }^{8}\right)$ The entropy as a function of temperature is recovered using $S(T)=\langle S(E)\rangle$ where the brackets denote the Boltzmann average over energy at $T$. The gradient $d S(E) / d E$ of the curves in Figure 2 represents the entropy cost of a favoured local structure. Here we see the fundamental influence of the choice of the FLS. Ranking the different FLS's in order of decreasing $d S(E) / d E$ we find

$$
\{0\}>\{31\}>\{22\}>\{33\} \approx\{32 c\}>\{23\} \approx\{24\}>\{32\}>\{1\} .
$$

This ordering of the entropy cost of adding an FLS among the different liquids is unchanged throughout the entire temperature range over which the liquid is the equilibrium state. This means that to account for this ordering it is sufficient to do so in the high temperature limit. In an empirical fit at high $T, S(E)=S\left(E_{\infty}\right)-A_{f i t}\left(E-E_{\infty}\right)^{2}$, the parameter $A_{f t}$ captures the observed ordering of the liquids (see Table I where the decrease in $A_{f i t}$ corresponds to the decrease in $\left.d S(E) / d E\right)$. In general, we find high symmetry structures such as $\{0\}$ and $\{31\}$ incur a higher entropy cost per FLS (and, hence, a rapid decrease in $S(E)$ with decreasing energy) than do structures with lower symmetry such as $\{32\}$ or $\{1\}$.

We would expect that the smaller the multiplicity $g$ of a FLS, the greater the entropy loss when one more FLS is added. As shown in Table I, however, $g$ provides a poor guide to the entropy cost of FLS's, as quantified by $A_{f i t}$. There are
TABLE I. Summary of the main results for each choice of FLS, organized in order of decreasing entropy cost from left to right, a trend accurately captured by $A_{\text {calc }}$. The fitting for $A_{f i t}$ was carried out over the range -0.005 $\leq E-E_{\infty} \leq 0$.

\begin{tabular}{lllllllllll}
\hline \hline FLS & $\{0\}$ & $\{31\}$ & $\{22\}$ & $\{33\}$ & $\{32 \mathrm{c}\}$ & $\{23\}$ & $\{24\}$ & $\{32\}$ & $\{1\}$ \\
\hline$g$ & 1 & 2 & 3 & 6 & 6 & 6 & 6 & 12 & 6 \\
$\begin{array}{l}\text { Freezing } \\
\text { temp. }\end{array}$ & 1.43 & 0.85 & 0.96 & 0.68 & 0.32 & 0.52 & 0.54 & 0.52 & 0.48 \\
$a \times 1728$ & 63 & 24 & 21 & 12 & 12 & 42 & 42 & 36 & 132 \\
$A_{\text {fit }}$ & 15.6 & 11.4 & 8.9 & 5.2 & 5.2 & 4.0 & 4.0 & 2.5 & 2.3 \\
$A_{\text {calc }}$ & 19.5 & 11.9 & 9.1 & 5.2 & 5.2 & 4.0 & 4.0 & 2.5 & 2.3 \\
\hline \hline
\end{tabular}

significant differences between FLS's with the same multiplicity $g=6$ (for which $A_{f i t}$ varies over a factor 2 ). Moreover, structure $\{1\}$ (with $g=6$ ) ranks below the less symmetric FLS $\{32\}$. We conclude that the entropic contribution from the interaction between FLS's is necessary to understand the correlation between geometry and entropy. To this end, we shall consider approximating $\Omega(E)$ with a Gaussian distribution (i.e., the second order Taylor expansion of the entropy $S(E)$ with respect to $E$ ). This approximation corresponds to counting the possible configurations of overlapping pairs of FLS's while neglecting higher order aggregates. If the density of states $\Omega(E)$ is expressed as

$$
\Omega(E)^{1 / N}=\rho_{o} \exp \left(-\frac{\left(E-E_{\infty}\right)^{2}}{2 \Lambda}\right)
$$

then our task is to evaluate the parameters $E_{\infty}, \rho_{o}$ and $\Lambda$. The first two terms are straightforward: $E_{\infty}=-g / 2^{6}$ and $\rho_{o}$, the total number of states per site, is just 2 . The parameter $\Lambda$ is directly related to the variance of the total energy of the system $\Xi=\sum_{i} E_{i}$ at infinite temperature (the sum here being over the lattice sites) through the relation $\Lambda=(1 / N)\left(\left\langle\Xi^{2}\right\rangle-\left(N E_{\infty}\right)^{2}\right)$. To make explicit the dependence of $\Lambda$ on the correlation between the energies on pairs of sites, we can expand the variance as

$$
\Lambda=\sum_{i} \sum_{j}\left(\left\langle E_{i} E_{j}\right\rangle-E_{\infty}^{2}\right)
$$
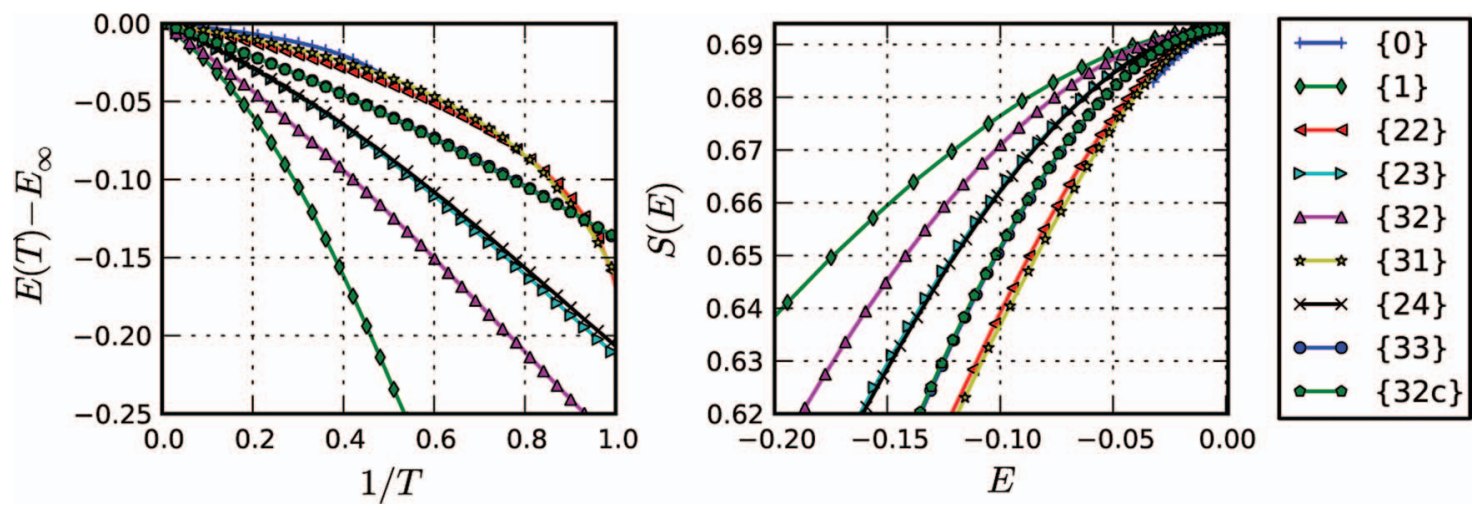

FIG. 2. Left panel: The potential energy/site $E(T)$ relative to $E_{\infty}$ as a function of $1 / T$ in the FLS model for all possible choices of the favoured local structure. Right panel: The entropy/site $S(E)$ as a function of the energy difference $E-E_{\infty}$. Note that in both plots the curves for $\{32 \mathrm{c}\}$ and $\{33\}$ are superimposed on one another. The $\{0\}$ curve is restricted to $T>2$ to avoid its freezing transition. 

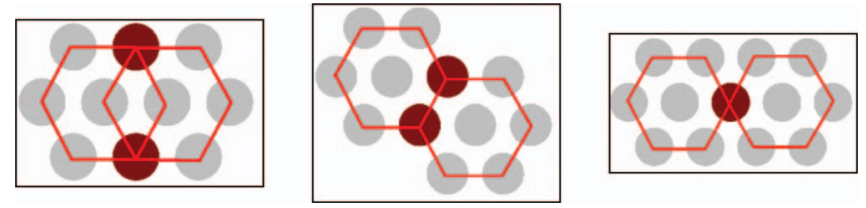

FIG. 3. Examples of the three distinct pairs of sites whose neighbourhoods overlap on the triangular lattice. Dark sites are shared by the local structures of both considered sites.

The product $E_{i} E_{j}$ equals zero unless both sites $i$ and $j$ are in the FLS, in which case the product equals 1. Therefore, $\left\langle E_{i} E_{j}\right\rangle$ is equal to the joint probability of $i$ and $j$ both having FLS neighbourhoods. Since we consider a random configuration uniformly distributed, $E_{i}$ and $E_{j}$ are independent if their structures do not overlap. There are three distinct types of overlap of the neighbourhoods of two sites and these are sketched in Figure 3. As each type of overlap has 6 different orientations, we have a total of 18 possible overlapping pairs to consider. The average value $\left\langle E_{i} E_{j}\right\rangle$ is

$$
\begin{aligned}
\left\langle E_{i} E_{j}\right\rangle & =p & & \text { if } i=j \\
& =p P(i \mid j) & & \text { if environments overlap. } \\
& =p^{2} & & \text { otherwise }
\end{aligned}
$$

Here $P(i \mid j)$ is the probability for site $i$ to be FLS, given that site $j$ is FLS. $p$ is the probability that a site is FLS so that $p=-E_{\infty} . P(i \mid j)$ reflects the fact that an FLS site exercises some constraint on the local structure of the neighbouring sites through the overlaps. A $\{0\}$ site, for example, completely surrounded by "up" spins, imposes that same spin alignment at the points of overlap with its neighbours and, in so doing, increases much the likelihood (over the random case) that these neighbouring sites will also be $\{0\}$. This tendency to aggregate, expressed as $P(i \mid j)>p$, is exhibited by all choices of FLS, to different degrees. $P(i \mid j)$ can be calculated by explicit enumeration (for each type of neighbourhood overlap shown in Figure 3) of the ratio of the number of spin configurations about the neighbouring sites that correspond to an overlapping pair of FLS over the total number of possible spin configurations. Let $a$ be the average of $(P(i \mid j)-p)$ over 18 neighbours of the three types of overlap. Then we can write

$$
\Lambda=p(1-p)+18 a p,
$$

where the exact value of $a$ for each FLS is provided in Table I. A large value of $a$ indicates that the FLS's tend to aggregate and form dimers. Two features of FLS geometry contribute to the magnitude of $a$. The multiplicity of the FLS establishes the number of distinct orientations a pair of FLS's could, in principle, adopt. Consider, for example, the large value of $a$ for the $\{1\}$ liquid. The 5 similar spins in this FLS allow for overlaps for many different orientations while the unique spin renders each of the FLS orientations distinct. The \{33\} FLS has the same number of distinct orientations as the $\{1\}$ FLS, but is considerably more constrained when it comes to overlapping with another $\{33\}$ site, hence the low value of $a$.

Within this approximation, the entropy is

$$
S(E)=\ln (2)-A_{\text {calc }}\left(E-E_{\infty}\right)^{2},
$$

where $A_{\text {calc }}=(2 \Lambda)^{-1}$ and $\langle E\rangle=E_{\infty}-\Lambda / T$. In Table I we compare the values of $A_{f i t}$ and $A_{\text {calc }}$ for the different choices of FLS and find excellent agreement. While the Gaussian approximation consistently underestimates the density of states, as shown in Figure 4, our theoretical expression successfully reproduces the ranking of the different liquids with respect to the entropy cost per FLS. This success includes differentiating the 5 local structures with 6-fold multiplicity. The two couples of FLS's that happen to have both same multiplicity g and same pair factor $a(\{23\}$ and $\{24\} ;\{33\}$ and $\{32 \mathrm{c}\})$ have $E(T)$ curves that superimpose almost perfectly in the whole liquid range, though the geometrical properties of these systems are apparently very different. This result establishes the fact that the entropic cost of a favoured local structure depends not only on the symmetry of the structure itself but also on the multiplicity of the aggregates of these structures.

In this paper, we have mapped out the connection between the geometry of the microscopic local structure that are favoured energetically and the density of states of the liquid. With the liquid's density of states, we can account for the observed behaviour of the energy, entropy, and heat capacity. This is, to our knowledge, the first demonstration of the relationship between the details of a locally preferred structure and macroscopic properties of the equilibrium liquid. The key features influencing this density of states are the multiplicity of the favoured structures, which is high for low symmetry structures, but also the multiplicity of their aggregates.
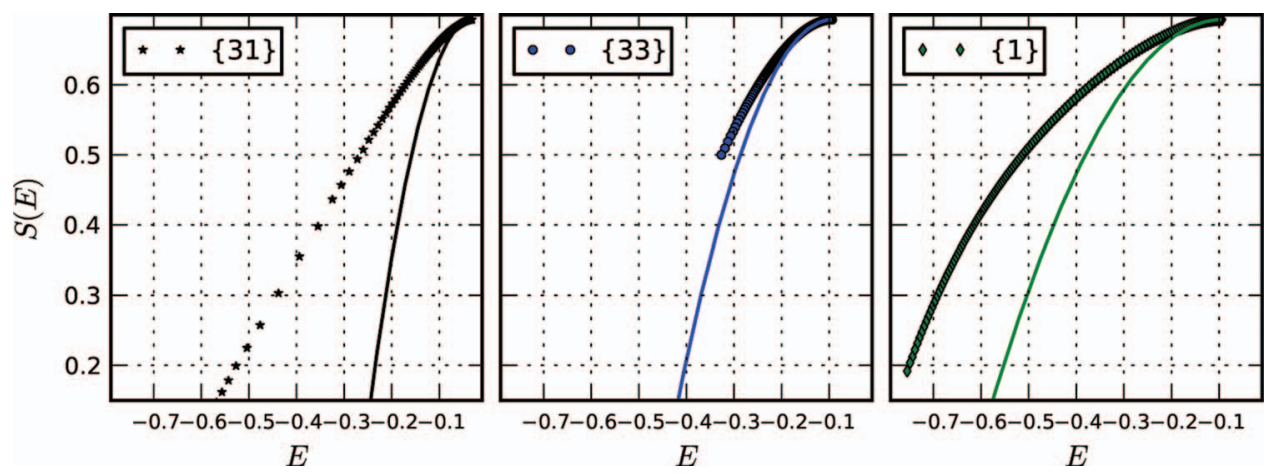

FIG. 4. Comparison of the entropy $S(E)$, calculated using Eq. (1) (points), with the quadratic approximation Eq. (7) (solid line). 
We arrive to the simple conclusion that liquids in which low energy structures can be inserted in a large number of ways due to low symmetry and loose geometrical constraints - are able to retain more entropy as their energy is made to decrease than the symmetric and more constrained ones. Consequently, they will show a higher heat capacity, a direct consequence of the greater accumulation of the favoured local structure in the equilibrium liquid on cooling. Extending these insights to entropic cost of local ordering in more realistic particle models of liquids presents us with a number of challenges. If the favoured local structures for a given Hamiltonian are to be identified by the frequency of their occurrence in a liquid then, in contrast to the FLS model, entropy, as well as energy, will be involved in that identification. In real liquids, the local structures would experience distortions whose influence on symmetry, entropy, and energy would need to be considered. While any model has its limits, we note that it is a simple task to include more than one FLS. Depending on the choice of combinations of FLS's, our lattice mode could provide some insight into the consequences of competition between or mutual aggregation of favoured local structures. A greater range and structural complexity of FLS's is also available with the extension of the lattice model to a high coordinated 3D lattice. Given the great potential utility of establishing a connection between local structure and the thermal properties of a liquid, we believe that these challenges are well worth addressing.

We acknowledge funding from the École Normale Suprieure and the Australian Research Council.

${ }^{1}$ P. H. Gaskell, J. Non-Cryst. Solids 357, 2769 (2011); K. Hiroshi and T. Hanyuki, J. Chem. Phys. 132, 104504 (2010).

${ }^{2}$ S. Mossa and G. Tarjus, J. Non-Cryst. Solids 352, 4847 (2006); D. Coslovich and G. Pastore, J. Chem. Phys. 127, 124504 (2007);

H. Tanaka, T. Kawasaki, H. Shintani, and K. Watanabe, Nature Mater. 9, 324 (2010).

${ }^{3}$ C. P. Royall, S. R. Williams, T. Ohtsuka, and H. Tanaka, Nature Mat. 7, 556 (2008).

${ }^{4}$ H. W. Sheng, et al., Nature (London) 439, 419 (2006); Y. Q. Cheng, E. Ma, and H. W. Sheng, Phys. Rev. Lett. 102, 245501 (2009).

${ }^{5}$ U. R. Pedersen, T. Schoeder, J. Dyre, and P. Harrowell, Phys. Rev. Lett. 104, 105701 (2010).

${ }^{6} \mathrm{P}$. Ronceray and P. Harrowell, Europhys. Lett. 96, 36005 (2011).

${ }^{7}$ A. B. Bortz, M. H. Kalos, and J. L. Lebowitz, J. Comp. Phys. 17, 10 (1975).

${ }^{8}$ A. Heuer, J. Phys.: Condens. Matter 20, 373101 (2008). 